\title{
The Glenolabral Articular Disruption Lesion Is a Biomechanical Risk Factor for Recurrent Shoulder Instability
}

\author{
Jens Wermers, M.Sc., Benedikt Schliemann, M.D., Michael J. Raschke, M.D., \\ Felix Dyrna, M.D., Lukas F. Heilmann, M.D., Philipp A. Michel, M.D., and \\ J. Christoph Katthagen, M.D.
}

\begin{abstract}
Purpose: To investigate the biomechanical effect of a glenolabral articular disruption (GLAD) lesion on glenohumeral laxity. Methods: Human cadaveric glenoids $(n=10)$ were excised of soft tissue, including the labrum to focus on the biomechanical effects of osteochondral surfaces. Glenohumeral dislocations were performed in a robotic test setup, while displacement forces and three-dimensional morphometric properties were measured. The stability ratio (SR), a biomechanical characteristic for glenohumeral stability, was used as an outcome parameter, as well as the path of least resistance, determined by a hybrid robot displacement. The impacts of chondral and bony defects were analyzed related to the intact glenoid. Statistical comparison of the defect states on SR and the path of least resistance was performed using repeated-measures ANOVA and Tukey's post hoc test for multiple comparisons $(P<.05)$. The relationship between concavity depth and SR was approximated in a nonlinear regression. Results: The initial SR of the intact glenoid $(28.3 \pm 7.8 \%)$ decreased significantly by $4.7 \pm 3 \%$ in case of a chondral defect $(P=.002)$. An additional loss of $3.2 \pm 2.3 \%$ was provoked by a $20 \%$ bony defect $(P=.004)$. The path of least resistance was deflected significantly more inferiorly by a GLAD lesion $\left(2.9 \pm 1.8^{\circ}, P=.002\right)$ and even more by a bony defect $\left(2.5 \pm 2.9^{\circ}, P=.002\right)$. The nonlinear regression with concavity depth as predictor for the SR resulted in a high correlation coefficient $(r=.81)$. Conclusions: Chondral integrity is an important contributor to the SR. Chondral defects as present in GLAD lesions may cause increased laxity, influence the humeral track on the glenoid during dislocation, and represent a biomechanical risk factor for a recurrent instability. Clinical Relevance: Cartilage deficiency corresponding to GLAD lesions may be a risk factor for impaired surgical outcomes.
\end{abstract}

\section{Introduction}

G lenohumeral stability is ensured by a variety of active and passive structures. Ligamentous and capsular restraints stabilize the joint during extreme movements. In the midrange of motion, these

From the University Hospital Münster, Department of Trauma, Hand, and Reconstructive Surgery, Münster, Germany.

The authors report the following potential conflicts of interest or sources of funding: B.S. reports personal fees from KLS Martin as a consultant, outside the submitted work. Full ICMJE author disclosure forms are available for this article online, as supplementary material.

Received April 6, 2021; accepted August 17, 2021.

Address correspondence to Jens Wermers, M.Sc., University Hospital Muenster, Department of Trauma, Hand, and Reconstructive Surgery, Waldeyerstraße 1,48149 Münster, Germany.E-mail: jens.wermers@ ukmuenster.de

(C) 2021 THE AUTHORS. Published by Elsevier Inc. on behalf of the Arthroscopy Association of North America. This is an open access article under the CC BY-NC-ND license (http://creativecommons.org/licenses/by-nc-nd/4.0/). 2666-061X/21515

https://doi.org/10.1016/j.asmr.2021.08.007 structures are lax, and stabilization is provided by compressing forces exerted by the rotator cuff. The humeral head is virtually pulled into the cavity formed by cartilage and labrum. This mechanism is called concavity-compression and is known as a main stabilizing effect of the glenohumeral joint. ${ }^{1-3}$

The bony glenoid is small and flat related to the articular surface of the humeral head. ${ }^{2}$ The curvature is enhanced by cartilage and labrum resulting in conforming glenohumeral articular surfaces. ${ }^{4}$ The labrum itself contributes to up to $50 \%$ of the glenoid depth..$^{5}$ A tear of the anteroinferior labrum (Bankart lesion) results in a reduced glenoid depth and an impaired concavity-compression. This is supported by several studies that proved labral tears to significantly affect glenohumeral stability. ${ }^{1-3,6,7}$ In these biomechanical studies, glenohumeral stability was commonly estimated by the evaluation of the stability ratio (SR). The SR results from the maximum dislocating force withstood by the joint compared to a predetermined medially aligned compression force. ${ }^{2,8}$ 
Loss of restraining structure in case of a Bankart lesion results in reduced force needed to dislocate the humeral head. Therefore, a resection of the labrum was shown to provoke a decrease of $10-20 \%$ in SR. ${ }^{2,7}$ The effect of a combined chondral-labral defect on glenohumeral stability was investigated by Lazarus et al., who revealed a loss of $65 \%$ in SR. ${ }^{3}$ A separate analysis of chondral integrity, and its effect on glenohumeral laxity, has not been investigated biomechanically yet. Patients with an isolated chondral defect often show no evidence of instability during physical examination or at surgery. ${ }^{9,10}$ However, a current biomechanical study proved that the concavity of the chondral surface is highly correlated with the SR. ${ }^{11}$ A chondral defect, thus, might be related to a loss in concavity and glenoid depth, as well as an enhanced glenohumeral laxity.

A clinical case of a chondral defect is the glenolabral articular disruption (GLAD) lesion. The GLAD lesion was first mentioned by Neviaser and was characterized as an anteroinferior cartilage injury adjacent to a superficial tear of the labrum. ${ }^{9}$ Since then, several variations of the GLAD lesion have been presented in sporadic case reports with different pathogenic mechanisms, but all have a cartilage defect in common. ${ }^{12-18}$ In a retrospective outcome study, Pogorzelski et al. detected a GLAD lesion as a potential risk factor for a recurrent shoulder instability after arthroscopic Bankart repair. The authors concluded that a GLAD lesion may be associated with as-yet-undetermined factors that predisposes patients to failure of an isolated Bankart repair. ${ }^{19}$ In this experimental study, the biomechanical effect of an anteroinferior chondral defect on the SR was analyzed. According to independent expert opinion, this is the most common location of GLAD lesions. The purpose was to investigate the biomechanical effect of a GLAD lesion on glenohumeral laxity. The hypothesis was that a GLAD lesion impairs chondral integrity and significantly reduces the displacement force that causes a dislocation.

\section{Methods}

\section{Ethical Approval}

All donors of human cadaveric specimen provided written consent to use their bodies for scientific and/or educational purposes. An approval of the institutional review board was obtained (IRB no. 2014-421-f-N).

\section{Preparation}

A total of 12 fresh-frozen human cadaveric scapulae were obtained from the University of Lübeck, Germany. Each specimen was thawed at room temperature and all soft tissue, including the labrum was removed. The labrum was dissected as its stabilizing effect had been analyzed in detail previously. ${ }^{2,3,7}$ In this study, however, the biomechanical effects of the

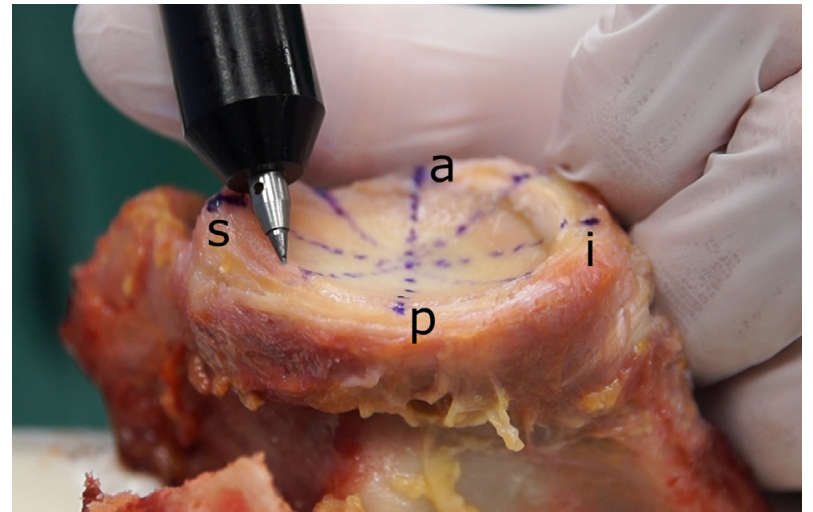

Fig 1. Digitization of the intact chondral articular glenoid surface. The long and short axes of the glenoid were aligned to the superoinferior (s-i) and anteroposterior (a-p) axes enabling the robot movement in a joint-specific coordinate system.

osteochondral surface were intended to be focused, and the loss of SR due to cartilage defects as in a GLAD lesion should be compared with the loss of SR due to bony defects. Because the labrum is also disrupted in bony glenoid defects, it was excluded from the comparison entirely. In addition, the acromion and coracoid were detached to avoid interference with the humeral head during dislocation.

Two cadaveric specimens suffered from osteoarthritis and were excluded from testing, resulting in a final sample size of $n=10$ specimen ( 3 right, 7 left; 5 males, 5 females; mean age $79.5 \pm 8.54$ [64-93] years) with macroscopically inconspicuous chondral surfaces. The scapulae were potted in polyurethane casting resin (RenCast PU, Gößl + Pfaff, Karlskron/Brautlach, Germany) and positioned in a robotic test setup, such that the glenoid plane, defined by the perpendicular long and short axes of the glenoid, was aligned horizontally to the floor. Artificial humeri (1028/1028-20, Sawbones, Malmö, Sweden) were potted and equipped with stemless shoulder implants (Eclipse, Arthrex, Munich, Germany). The trial heads of this implant were used to adapt for size and radius of each glenoid. Therefore, two surgeons selected the trial heads according to a best-fit approach and their clinical experience by choosing the smallest head that was still large enough to completely cover the glenoid cavity. In addition, the implant provided a standardized interface without cartilage or bony defects of the human cadaveric humeri.

\section{Test Setup}

An industrial robot (KR 60-3, KUKA, Augsburg, Germany) with 6 degrees of freedom (DOF) was used to perform translations of the implanted artificial humeri in the cadaveric glenoids with a position repeatability of .06 $\mathrm{mm}$. Translational forces were measured with an 
Fig 2. Dislocation of the humeral head in a right glenoid for a pure anterior translation (A) and for an anterior translation with continuous minimization of superoinferior forces (B). By minimizing superoinferior (s-i) forces, the path of least resistance was determined.

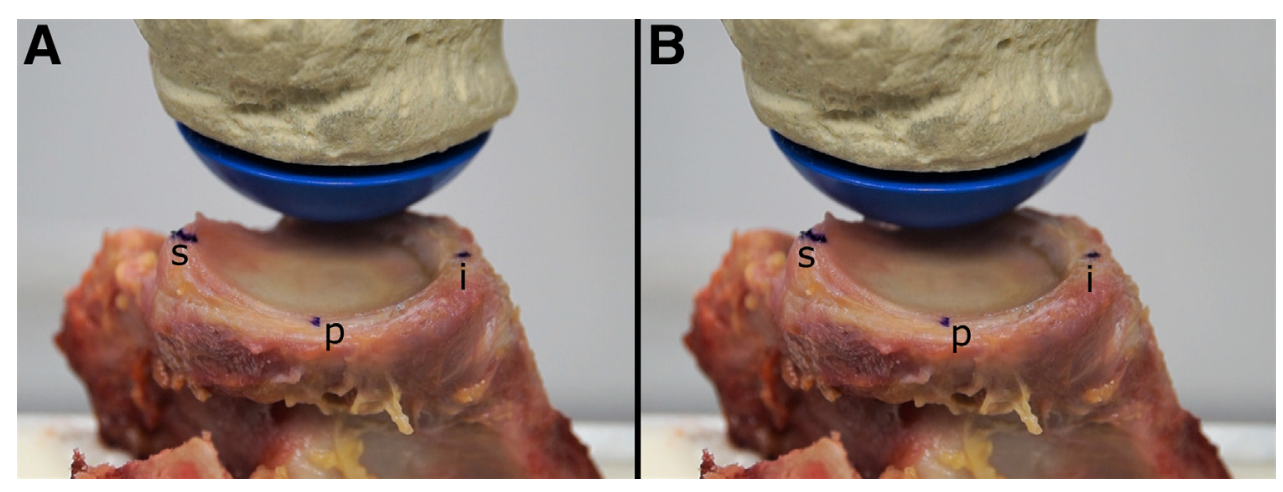

accuracy of .25 N using a force torque sensor (Mini45, ATI Industrial Automation, Apex, NC) mounted between the humeri and the robot. The intact chondral surface of the glenoids was captured using a threedimensional (3D) measuring arm (Absolute Arm 83207, Hexagon Metrology, Wetzlar, Germany) as shown in Fig 1. A total of more than 100 surface points were digitized to evaluate glenoid length and width corresponding to the long and short axes of the glenoid, as well as the glenoid concavity. The error of 3D measurements was smaller than $.05 \mathrm{~mm}$. In addition, these measurements were deployed to set up a joint specific coordinate system for each glenoid. Anatomical landmarks of the glenoid and artificial humerus were chosen to align the superoinferior and anteroposterior axes onto the long and short axes of the glenoid, respectively. Translations of the humeral head were performed using a software for robotic joint testing (simVITRO, Cleveland Clinic BioRobotics Lab, Cleveland, $\mathrm{OH}$ ) within these calibrated axes, thus minimizing the effects of a physiological glenoid version.

\section{Experiments}

The humeral head was positioned in glenohumeral abduction of $56^{\circ}$ and neutral rotation. This position corresponds to $90^{\circ}$ humerothoracic elevation and represents the midrange of motion where concavitycompression is known to be a main stabilizer of the glenohumeral joint. ${ }^{1,20}$ A neutral position of the humeral head inside the glenoid cavity was found by application of $50 \mathrm{~N}$ medial compression force exerted through the robot and minimizing all other force components. The value of $50 \mathrm{~N}$ was chosen in correspondence to several biomechanical studies focusing on the SR, as $50 \mathrm{~N}$ was reported with no chondral deformation over 30 minutes and no gross damage to the glenoid during dislocation. ${ }^{2,3}$ This compression force was maintained continuously in all experiments and used for the calculation of SR.

The robot was controlled to perform two types of dislocation: 1) a pure anterior dislocation parallel to the short axes, according to the $3 \mathrm{o}^{\prime}$ clock direction related to a right glenoid (Fig 2A) and 2) an anterior dislocation, while continuously minimizing superoinferior forces (Fig 2B). During the second, hybrid dislocation, the humeral head was able to move not only anterior, but also superior or inferior depending on where the least forces occur. This displacement was performed to identify the path of least resistance and to determine a shift in this path caused by a chondral or bony defect. The displacement rate was regulated to a maximum of $1 \mathrm{~mm} / \mathrm{s}$ for each type of dislocation and direction of displacement.

Both dislocation types were performed once for each of three defect states: 1) intact glenoid, 2) GLAD lesion, and 3) $20 \%$ bone defect. To simulate a GLAD lesion, a chondral defect was created anteroinferior, extending from $3 \mathrm{o}^{\prime}$ clock to $4: 30$ relative to a right glenoid and a width of $20 \%$ relative to the intact glenoid width (Fig 3 , A-C). In this spot, the cartilage was removed down to the subchondral bone surface. Currently, there is little scientific background regarding incidence and location of the chondral defect. Therefore, we obtained independent expert opinion from three internationally well-known senior surgeons, resulting in the chosen characteristic location of a GLAD lesion. Afterwards, the $20 \%$ bone defect was created with a hand-guided rotary tool (Multitool 4000, Dremel, Breda, Netherlands) parallel to the long axis of the glenoid (Fig 3, D and E). The defect line was digitized with the 3D measuring arm for an evaluation of the true defect width as a percentage of the intact glenoid width.

Measurements of the load cell were used to determine the SR by dividing the maximum resultant anterior force by the constant compression force of $50 \mathrm{~N}$. The displacement of the humeral head during dislocation was acquired from the position data of the robot. The lateral displacement for a pure anterior dislocation is shown in Fig 4 for all defect states. The maximum lateral displacement equals the concavity depth (d), as previously described by Moroder et al. ${ }^{21}$ For the hybrid dislocation, the path of least resistance performed by the robot was calculated from the robot position data. As the robot positions were given as inferior and 
A

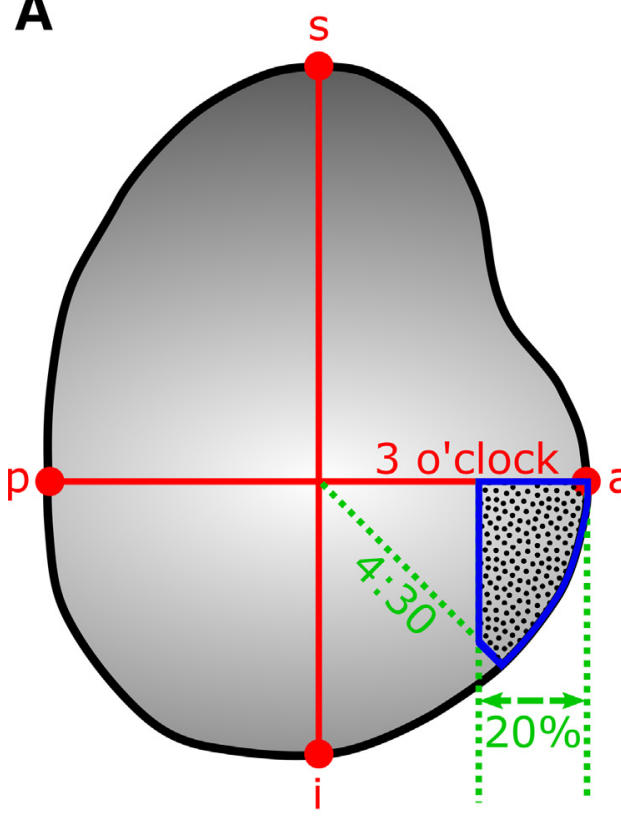

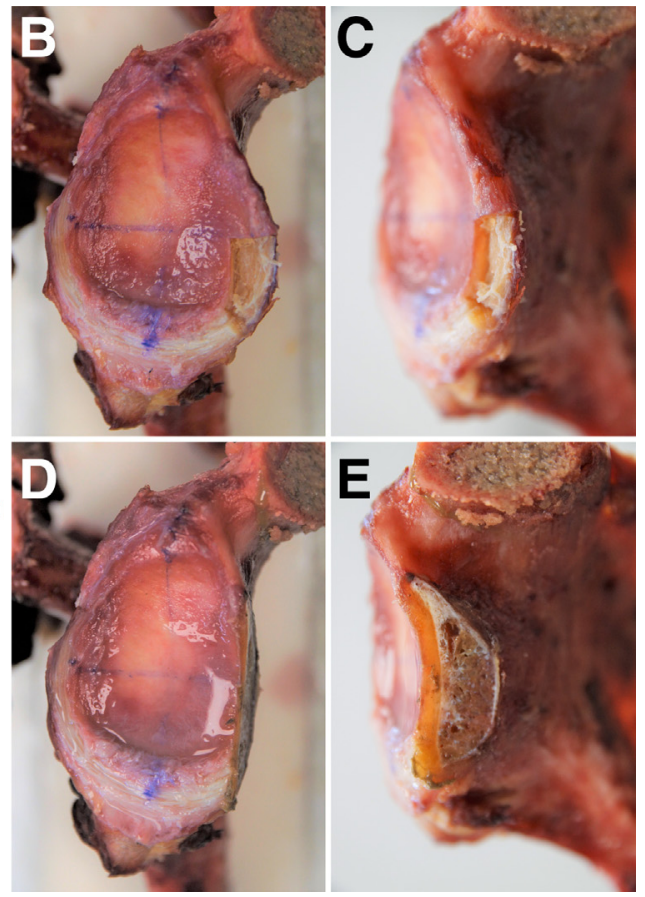

Fig 3. Schematic drawing of glenoid axes and chondral defect (A) and photographic images of the created chondral (B and $\mathrm{C}$ ) and bony (D and $\mathrm{E}$ ) defects. The chondral defect (dotted area in A) was located anteroinferior between 3 o'clock and 4:30 for right glenoids with a defect width of $20 \%$. The bony defect of $20 \%$ width was created parallel to the long axis of the glenoid, according to the superoinferior axis. anterior displacement separately, the inverse tangent of inferior related to anterior displacement was computed. This resulted in a directional angle that was evaluated at the point of maximum lateral displacement of the humeral head. Thereby, a change in dislocation direction due to cartilage and bony defects could be identified.

\section{Statistics}

Signal processing was performed with a custom-made MATLAB-Script (R2019a, The MathWorks, Natick, MA). The influence of each defect state (intact, GLAD lesion, and $20 \%$ bone defect) on the SR and on the path of least resistance was evaluated statistically in GraphPad Prism 9 (GraphPad Software, San Diego, CA). For these group comparisons, one-way repeated-measures ANOVA and Tukey's post hoc test with a correction for multiple comparisons were chosen along with a significance level of $P<.05$. In addition, the relationship between concavity depth $(d)$ and SR was analyzed for all defect states in a nonlinear regression model. The results are presented as means \pm SE of the mean and $95 \%$ confidence intervals (CI). On the basis of pretests and comparable studies focusing on the $\mathrm{SR}^{2,3,7}$ an effect size of $d_{\mathrm{z}}=1.2$ was assumed, resulting in a total sample size of $n=10$ to achieve a statistical power of .95 .

\section{Results}

The SR was significantly affected by a chondral defect, as well as by a $20 \%$ bone defect. The initial SR resulted in $28.3 \pm 2.5 \%$ (CI: 22.7 to $33.9 \%$ ), whereas a chondral defect led to a reduced SR of $23.6 \pm 2.1 \%$ (CI: 18.8 to
$28.4 \%$ ). A bony defect of $20 \%$ width provoked an additional loss of displacement force resulting in a mean SR of $20.4 \pm 1.7 \%$ (CI: 16.6 to $24.2 \%$ ). Both effects were significant with $P=.002$ and $P=.004$, respectively. The mean loss of SR of $7.9 \%$ was, therefore, composed of $4.7 \%$ loss by a chondral defect and additional $3.2 \%$ loss by a bony defect. These effects of a chondral or bony defect on the SR are shown in Fig 5 . The path of least resistance for dislocation was deflected inferiorly by $2.9 \pm .6^{\circ}$ (CI: 1.6 to $4.2^{\circ}$ ) in case of a chondral defect. A $20 \%$ bone defect caused an additional inferior shift by $2.5 \pm .9^{\circ}$ (CI: .4 to $4.6^{\circ}$ ), resulting in a rather anteroinferior dislocation of the humeral head. This effect was identified as significant with $P=.002$ for both defect states, while there was no significant difference between the direction of a chondral defect compared with a bony defect $(P=.06)$.

The nonlinear regression of SR in dependence on the concavity depth $(d)$ followed an exponential function, which is depicted in Fig 6. The correlation coefficient for this approximation was $r=.81$ and the mean squared error (MSE) resulted in $4.4 \%$, both indicating a high goodness of fit for the nonlinear regression. Because of this exponential behavior, the SR drops rapidly especially for small concavity depths below $1 \mathrm{~mm}$.

\section{Discussion}

The results of this experimental study can be concluded in two main findings: 1) A deficiency of the cartilage corresponding to a GLAD lesion leads to a reduced concavity depth and a significant loss of SR. 2) 
Fig 4. Lateral displacement of the humeral head for a pure anterior dislocation dependent on defect state; The concavity depth (d) is marked for the intact state and results from the maximum lateral displacement for each defect state.

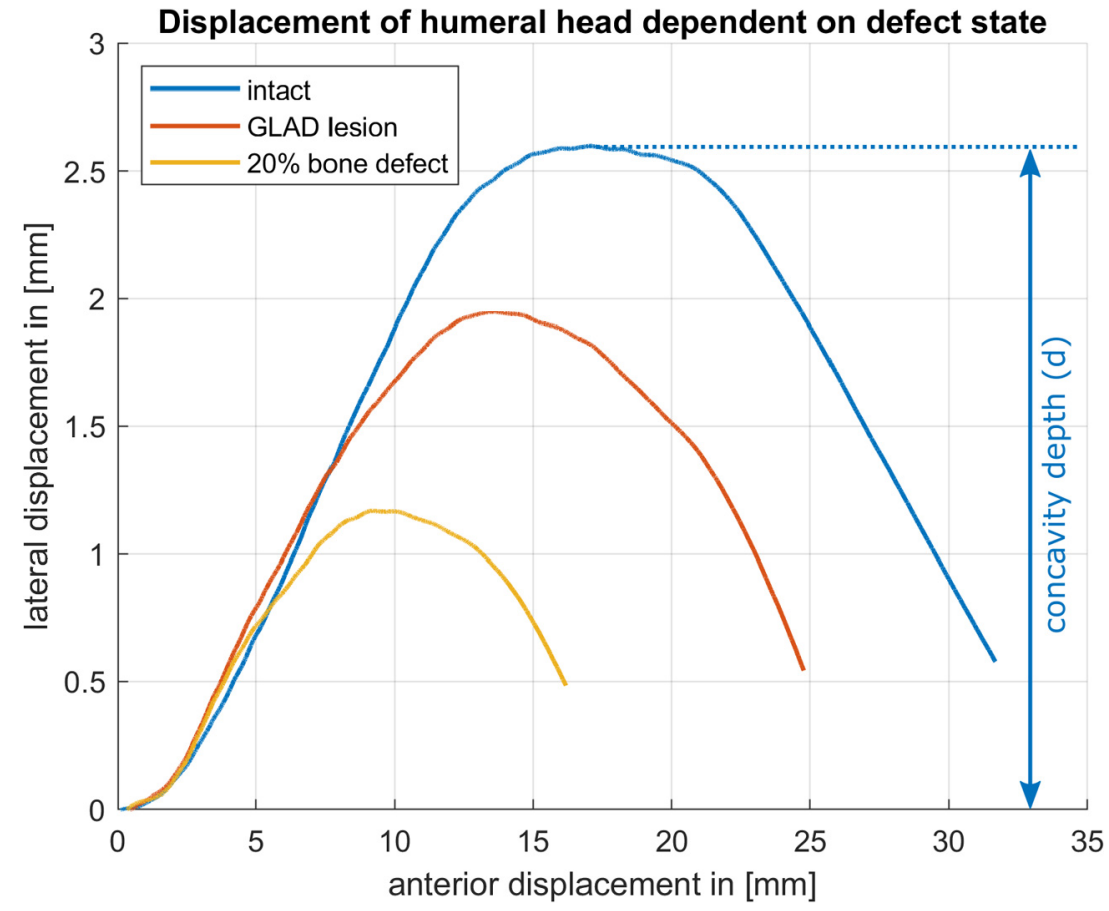

Anteroinferior chondral defects significantly influence the humeral track on the glenoid during dislocation in a more inferior direction.

To date, the GLAD lesion has been poorly explored and rarely considered as a risk factor for a recurrent instability. This was widely accepted since the GLAD lesion was characterized by Neviaser. ${ }^{9}$ Nevertheless, the GLAD lesion can be a persistent source of shoulder pain. ${ }^{16,22,23}$ As detection of chondral defects by MR arthrography is moderate, GLAD lesions are often identified only during arthroscopic treatment. ${ }^{12}$ Consequently, the treatment method is also usually based on arthroscopy, and it is conceivable that knowledge of biomechanical dependencies may help to optimize the clinical outcome. However, there are little data available about the biomechanical influence of

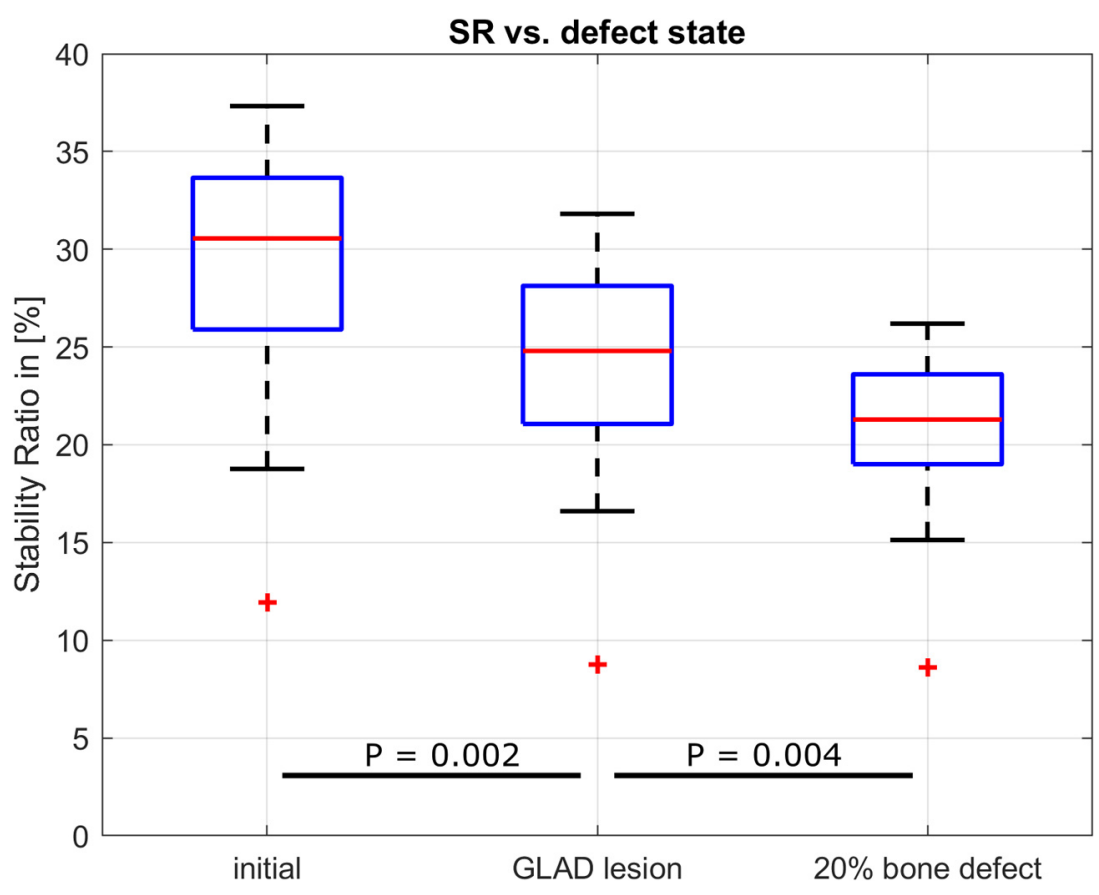

Fig 5. SR in dependence of defect state. The SR drops significantly by a chondral defect corresponding to a GLAD lesion and again significantly by a $20 \%$ bone defect. The red central marks indicate the median value. One specimen was identified as an outlier $(+)$. 


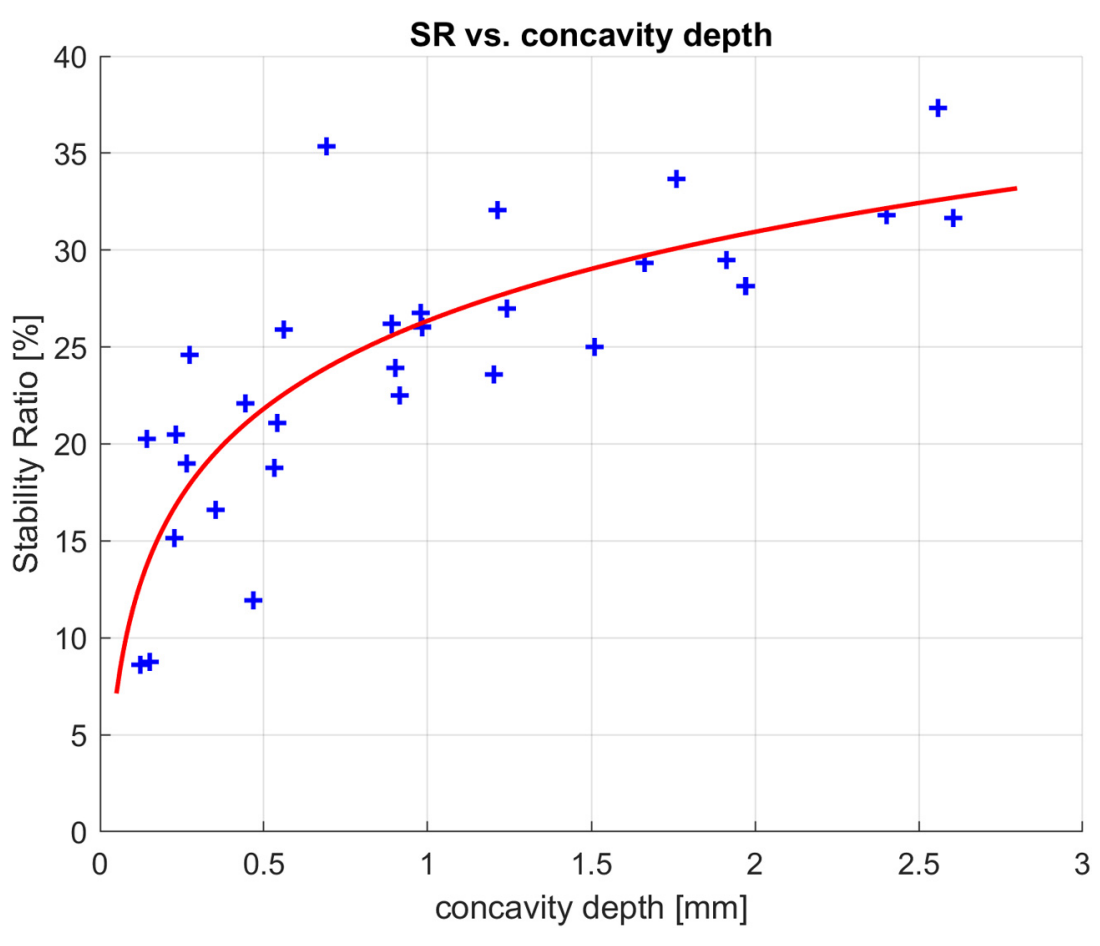

Fig 6. Nonlinear regression with concavity depth (d) as predictor for the SR. The markers indicate the discrete outcome measurements of all specimen and defect states, the red line shows the nonlinear approximation. The smaller the concavity depth, the faster the SR drops to a minimum. chondral defects, such as those present in a GLAD lesion, on glenohumeral laxity.

Two clinical studies recently analyzed clinical outcomes after arthroscopic Bankart repair (ABR). Pogorzelski et al. identified significant higher failure rates after ABR for patients with a GLAD lesion. ${ }^{19}$ They referred to as-yet-undetermined factors that could predispose these patients to failure. Davey et al. had a closer look on this assumption and analyzed the effect of a GLAD lesion by means of other patient-reported outcome scores. ${ }^{24}$ They identified no significant impact of a GLAD lesion after ABR. However, patients with a GLAD lesion showed a mean subjective shoulder value that was 8 points lower compared with controls. The fact that no significant difference was observed may be explained by missing power to detect a potentially clinically relevant difference. To date, knowledge of the clinical impact of a GLAD lesion is very limited and not yet consistently explored.

The SR, a biomechanical parameter for stabilization by concavity-compression, was often considered to be linearly dependent on concavity depth or bone loss. 2,3,25,26 However, Moroder et al. revealed a nonlinear dependency in their simulation-based studies by means of the bony shoulder stability ratio (BSSR). ${ }^{21,27}$ The BSSR is a mathematical approximation of the SR based on morphometric properties of the glenoid. This measure was derived to determine the stabilizing effect by the bony structure. By use of various theoretical combinations of joint radius and concavity depth, they revealed a nonlinear impact of concavity depth on the BSSR. These theoretical findings were confirmed in this experimental study. A certain loss of concavity depth may have a higher impact on the SR, the smaller the initial concavity depth. Especially for flat glenoids with a concavity depth below 1 $\mathrm{mm}$, the SR can rapidly drop to a minimum, even with small disturbances in concavity depth. However, it is important to note that apart from concavity depth, there are other characteristics that are linearly related to the SR. In a recent study focusing on osteochondral glenoid concavity, a highly linear correlation was demonstrated between the measured SR and the concavity gradient, as well as the geometrical based BSSR. ${ }^{11}$ The results of this study are, therefore, not contradictory but support the theory that a certain defect size can have a different influence on the SR depending on glenoid concavity and depth.

In this study, chondral integrity was also found to be highly relevant to the SR. The loss of SR due to a chondral defect was approximately half as much, as the loss caused by a $20 \%$ bony defect. In fact, in case of a GLAD lesion with a mostly intact labrum, the labrum increases the concavity depth and provides further stability. ${ }^{2,5,7}$ However, as the cartilage is no longer capable to absorb occurring dislocation forces, it is conceivable that the labrum is subject to greater stress. In addition, the labrum is not only much softer than cartilage, but also suffers a superficial tear in a GLAD lesion and may be weakened consequently. Thus, the results suggest a redistribution of displacement forces to the labrum and an increased risk of instability, although this needs to be explored in more detail through further biomechanical studies. 
Furthermore, a significant shift in the path of least resistance was detected, favoring the humeral dislocation more inferiorly toward the direction of the anteroinferior defect. As recently shown, the anatomy of the coracoid also has an influence on the deflection of the humeral head and the location of associated bony glenoid defects. ${ }^{28}$ This suggests that the path of least resistance may influence the location with higher risk for traumatic fractures. Thus, an anteroinferior located GLAD lesion may cause the humeral head to displace more inferiorly, where the coracoid may provide less or no bony restraint. Although the labrum contributes to glenohumeral stability by a large amount in case of a GLAD lesion, this shift of dislocation direction and incapability, and incapability of cartilage to restrain occurring displacement forces represents a biomechanical risk factor for recurrent instability.

The investigated biomechanical effects provide new insights in the change in direction of dislocation and reinforce the important role of concavity and chondral integrity. Over the last years, a variety of techniques for management of articular cartilage defects were evolved. ${ }^{29,30}$ In general, treatment depends on location, size, and depth of the chondral lesion, as well as concurrent pathology. ${ }^{31}$ Among all procedures, microfracture in combination with an advancement of capsulolabral tissues into the defect area has become a popular method not only due to excellent outcomes, but also due to a fast treatment. From a biomechanical point of view, it is important to address the cartilage loss by infilling the defect area to compensate for the loss of concavity and SR. The advancement of capsulolabral tissues could help to increase concavity depth and to avoid a shift in the path of least resistance to a more anteroinferior location, where the coracoid does not provide bony restraint.

\section{Limitations}

A major limitation of this study is that all soft tissues, including the labrum, as well as the coracoid process, were dissected. This was done to focus on the osteochondral related amount of laxity and to achieve a comparison between loss of SR due to cartilage and bony defects. In fact, the labrum provides additional stabilization in a clinical situation that may be sufficient to prevent from glenohumeral instability. However, the high loss of restraint and SR due to a cartilage defect as present in a GLAD lesion, and the change of dislocation direction represents a biomechanical risk factor for recurrent instability that may be relevant for clinical decision-making in the future. Another limitation is that humeral head implants were used to avoid preexisting chondral damage or incongruent spherical surfaces due to a high mean age of the specimen. It follows that only osteochondral defects at the glenoid, and not at the humeral head, were considered. Effects of humeral head defects such as a Hill-Sachs-lesion and on- or off-track defects remain unaddressed. A further limitation is the high mean age of the specimen, which does not represent the typical patient group of young athletics suffering from a GLAD lesion. However, specimens with visible signs of cartilage damage and osteoarthritis were excluded from testing. In addition, cartilage is typically more distinctive in younger patients, meaning that the influence of a GLAD lesion and importance of chondral integrity may be even higher in these patients. Despite the above limitations, the understanding of the relevance of chondral integrity and the biomechanical effects of a GLAD lesion on the glenohumeral joint has, therefore, improved.

\section{Conclusions}

Chondral integrity is an important contributor to the SR. Chondral defects as present in GLAD lesions may cause increased laxity, influence the humeral track on the glenoid during dislocation, and represent a biomechanical risk factor for a recurrent instability.

\section{Acknowledgments}

The authors acknowledge Peter J. Millett, M.D., Robert A. Arciero, M.D., and Markus Scheibel, M.D., for their expert opinion in extent and location of typical GLAD lesion injuries.

\section{References}

1. Lippitt S, Matsen F. Mechanisms of glenohumeral joint stability. Clin Orthop Relat Res 1993;291:20-28.

2. Lippitt SB, Vanderhooft JE, Harris SL, Sidles JA, Harryman DT, Matsen FA. Glenohumeral stability from concavity-compression: A quantitative analysis. J Shoulder Elb Surg 1993;2:27-35.

3. Lazarus MD, Sidles JA, Harryman DT, Matsen FA. Effect of a chondral-labral defect on glenoid concavity and glenohumeral stability. A cadaveric model. J Bone Joint Surg 1996;78:94-102.

4. Soslowsky LJ, Flatow EL, Bigliani LU, Mow VC. Articular geometry of the glenohumeral joint. Clin Orthop Relat Res 1992;285:181-190.

5. Howell SM, Galinat BJ. The glenoid-labral socket. A constrained articular surface. Clin Orthop Relat Res 1989;243:122-125.

6. Itoi E, Lee S-B, Berglund LJ, Berge LL, An K-N. The effect of a glenoid defect on anteroinferior stability of the shoulder after Bankart repair: A cadaveric study. J Bone Joint Surg 2000;82:35-46.

7. Halder AM, Kuhl SG, Zobitz ME, Larson D, An KN. Effects of the glenoid labrum and glenohumeral abduction on stability of the shoulder joint through concavitycompression. J Bone Joint Surg 2001;83:1062-1069.

8. Di Giacomo G, Piscitelli L, Pugliese M. The role of bone in glenohumeral stability. EFORT Open Rev 2018;3:632-640.

9. Neviaser TJ. The GLAD lesion: Another cause of anterior shoulder pain. Arthroscopy 1993;9:22-23. 
10. Sanders TG, Tirman PF, Linares R, Feller JF, Richardson R. The glenolabral articular disruption lesion: MR arthrography with arthroscopic correlation. Am J Roentgenol 1999;172:171-175.

11. Wermers J, Schliemann B, Raschke MJ, et al. Glenoid concavity has a higher impact on shoulder stability than the size of a bony defect. Knee Surg Sports Traumatol Arthrosc 2021;29:2631-2639.

12. Porcellini G, Cecere AB, Giorgini A, Micheloni GM, Tarallo L. The GLAD lesion: Are the definition, diagnosis and treatment up to date? A Systematic Review. Acta Biomed 2020;91:e2020020.

13. Agarwalla A, Puzzitiello RN, Leong NL, Forsythe B. Concurrent primary repair of a glenoid labrum articular disruption and a Bankart lesion in an adolescent: A case report of a novel technique. Case Rep Orthop 2019;2019: $1-6$.

14. Page R, Bhatia DN. Arthroscopic repair of a chondrolabral lesion associated with anterior glenohumeral dislocation. Knee Surg Sports Traumatol Arthrosc 2010;18:1748-1751.

15. Galano GJ, Weisenthal BM, Altchek DW. Articular shear of the anterior-inferior quadrant of the glenoid: A glenolabral articular disruption lesion variant. Am J Orthop 2013;42:41-43.

16. Zhu W, Lu W, Zhang L, et al. Arthroscopic findings in the recurrent anterior instability of the shoulder. Eur J Orthop Surg Traumatol 2014;24:699-705.

17. Singh RB, Hunter JC, Smith KL. Mri of shoulder instability: State of the art. Curr Probl Diagn Radiol 2003;32:127-134.

18. Lederman ES, Flores S, Stevens C, Richardson D, Lund P. The glenoid labral articular teardrop lesion: A chondrolabral injury with distinct magnetic resonance imaging findings. Arthroscopy 2018;34:407-411.

19. Pogorzelski J, Fritz EM, Horan MP, Katthagen JC, Provencher MT, Millett PJ. Failure following arthroscopic Bankart repair for traumatic anteroinferior instability of the shoulder: Is a glenoid labral articular disruption (GLAD) lesion a risk factor for recurrent instability? J Shoulder Elb Surg 2018;27:e235-e242.

20. Ludewig PM, Phadke V, Braman JP, Hassett DR, Cieminski CJ, LaPrade RF. Motion of the shoulder complex during multiplanar humeral elevation. J Bone Joint Surg 2009;91:378-389.

21. Moroder P, Ernstbrunner L, Pomwenger W, et al. Anterior shoulder instability is associated with an underlying deficiency of the bony glenoid concavity. Arthrosc $J$ Arthrosc Relat Surg 2015;31:1223-1231.

22. Hintermann B, Gächter A. Arthroscopic findings after shoulder dislocation. Am J Sports Med 1995;23:545-551.

23. Gross CE, Chalmers PN, Chahal J, et al. Operative treatment of chondral defects in the glenohumeral joint. Arthroscopy 2012;28:1889-1901.

24. Davey MS, Hurley ET, Colasanti CA, et al. Clinical outcomes of patients with anterior shoulder instability and glenolabral articular disruption lesions: A retrospective comparative study. Am J Sports Med 2020;48:3472-3477.

25. Gottschalk LJ, Walia P, Patel RM, et al. Stability of the glenohumeral joint with combined humeral head and glenoid defects. Am J Sports Med 2016;44:933-940.

26. Shin S-J, Ko YW, Scott J, McGarry MH, Lee TQ. The effect of defect orientation and size on glenohumeral instability: A biomechanical analysis. Knee Surg Sports Traumatol Arthrosc 2016;24:533-539.

27. Moroder P, Damm P, Wierer G, et al. Challenging the current concept of critical glenoid bone loss in shoulder instability: Does the size measurement really tell it all? Am J Sports Med 2019;47:688-694.

28. Li MM, Goetti P, Sandman E, Rouleau DM. Influence of coracoid anatomy on the location of glenoid rim defects in anterior shoulder instability: 3D CT-scan evaluation of 51 patients. Surg Radiol Anat 2020;42:895-901.

29. Elser F, Braun S, Dewing CB, Millett PJ. Glenohumeral joint preservation: Current options for managing articular cartilage lesions in young, active patients. Arthrosc J Arthrosc Relat Surg 2010;26:685-696.

30. Seidl AJ, Kraeutler MJ. Management of articular cartilage defects in the glenohumeral joint. J Am Acad Orthop Surg 2018;26:e230-e237.

31. Steinmetz G, Hamilton J, Fernandes C, Bond J. Matrixinduced autologous chondrycte implantation for a glenoid chondral defect. JBJS Case Connect 2020;10:e0264e0264. 